\title{
Maternal Serum Lipid, Estradiol, and Progesterone Levels in Pregnancy, and the Impact of Placental and Hepatic Pathologies
}

\author{
Mütterlicher Lipid-, Estradiol- und Progesteronspiegel in der Schwangerschaft \\ in Abhängigkeit von Plazenta- und Leberpathologien
}

Authors

Affiliations
U. Pecks ${ }^{1,3}$, W. Rath ${ }^{3}$, N. Kleine-Eggebrecht ${ }^{1}$, N. Maass ${ }^{3}$, F. Voigt ${ }^{1}$, T. W. Goecke ${ }^{1}$, M. G. Mohaupt ${ }^{2}$, G. Escher ${ }^{2}$

${ }^{1}$ Department of Obstetrics and Gynecology, University Hospital of the RWTH, Aachen, Germany

${ }^{2}$ Department of Nephrology, Hypertension and Clinical Pharmacology and Department of Clinical Research, Inselspital

University Hospital Bern, Bern, Switzerland

3 Department of Obstetrics and Gynecology, University Hospital Schleswig-Holstein, Kiel, Germany

Key words
○ pregnancy
O steroids
- lipids
— IUGR
- preeclampsia
Schlüsselwörter
O Schwangerschaft
- Steroide
- Lipide
- intrauterine Wachstums-
retardierung (IUGR)
- Präeklampsie

received 11.9.2015

revised $\quad 5.4 .2016$

accepted 20.4.2016

\section{Bibliography}

Dol http://dx.doi.org/

$10.1055 / \mathrm{s}-0042-107078$

Geburtsh Frauenheilk 2016; 76:

799-808 ๑ Georg Thieme

Verlag KG Stuttgart · New York ISSN 0016-5751

\section{Correspondence \\ Dr. U. Pecks}

Universitätsklinikum Schleswig-

Holstein Campus Kiel

Klinik für Gynäkologie

und Geburtshilfe

Arnold-Heller-Straße 3

24105 Kiel

Germany

upecks@ukaachen.de

\section{Abstract}

$\nabla$

Objective: Lipids and steroid hormones are closely linked. While cholesterol is the substrate for (placental) steroid hormone synthesis, steroid hormones regulate hepatic lipid production. The aim of this study was to quantify circulating steroid hormones and lipid metabolites, and to characterize their interactions in normal and pathological pregnancies with a focus on hepatic and placental pathologies.

Methods: A total of 216 serum samples were analyzed. Group A consisted of 32 patients with uncomplicated pregnancies who were analyzed at three different time-points in pregnancy (from the first through the third trimester) and once post partum. Group B consisted of 36 patients (24th to 42nd week of gestation) with pregnancy pathologies (IUGR $n=10$, preeclampsia $n=13$, HELLP $n=6$, intrahepatic cholestasis $n=7$ ) and 31 controls with uncomplicated pregnancies. Steroid profiles including estradiol, progesterone, and dehydroepiandrosterone were measured by GC-MS and compared with lipid concentrations.

Results: In Group A, cholesterol and triglycerides correlated positively with estradiol (cholesterol $\rho=0.50$, triglycerides $\rho=0.57$ ) and progesterone $(\rho=0.49, \rho=0.53)$ and negatively with dehydroepiandrosterone $(\rho=-0.47, \rho=-0.38)$. Smoking during pregnancy affected estradiol concentrations, leading to lower levels in the third trimester compared to non-smoking patients $(\mathrm{p}<0.05)$. In Group B, cholesterol levels were found to be lower in IUGR pregnancies and in patients with HELLP syndrome compared to controls $(p<0.05)$. Steroid hormone concentrations of estradiol $(p<0.05)$ and progesterone $(\mathrm{p}<0.01)$ were lower in pregnancies with IUGR.

Discussion: Lipid and steroid levels were affected most in IUGR pregnancies, while only minor changes in concentrations were observed for other pregnancy-related disorders. Each of the ana-

\section{Zusammenfassung \\ $\nabla$}

Zielsetzung: Lipide und Steroidhormone stehen in wechselseitiger Beziehung zueinander. Cholesterin ist Ausgangssubstanz für die (plazentare) Steroidhormonsynthese. Die Lipidproduktion in der Leber wird von Steroidhormonen reguliert. Ziel der Studie war es, die Konzentration von Steroidhormonen und Lipiden im Blut im Verlauf der Schwangerschaft und bei Schwangerschaftspathologien mit Beteiligung von Leber oder Plazenta zu quantifizieren.

Methoden: 216 Serumproben wurden analysiert. Gruppe A bestand aus 32 Patientinnen mit unkomplizierter Schwangerschaft, denen zu drei Zeitpunkten im Schwangerschaftsverlauf sowie einmal postpartal Blut abgenommen wurde. Gruppe B bestand aus 36 Patientinnen (24.-42. SSW) mit Schwangerschaftspathologien (intrauterine Wachstumsrestriktion [IUGR] $n=10$, Präeklampsie $n=13$, HELLP-Syndrom $n=6$, intrahepatische Cholestase $n=7$ ) sowie 31 Frauen mit unkomplizierter Schwangerschaft. Ein Steroidprofil einschließlich Estradiol, Progesteron und Dehydroepiandrosteron wurde mit der Gaschromatografie-Massenspektrometrie (GC-MS) erstellt und mit Lipidkonzentrationen verglichen. Ergebnisse: In der Gruppe A korrelierten Cholesterin- und Triglyzeridwerte positiv mit Estradiol (Cholesterin $\rho=0,50$, Triglyzeride $\rho=0,57$ ) und Progesteron $(\rho=0,49, \rho=0,53)$ und negativ mit Dehydroepiandrosteron $(\rho=-0,47, \rho=-0,38)$. Rauchen während der Schwangerschaft führte zu niedrigeren Estradiolkonzentrationen im 3. Trimenon im Vergleich zu Nichtrauchern $(p<0,05)$. In der Gruppe B wies die IUGR-Gruppe deutlichste Veränderungen auf. Hier zeigten sich verglichen mit der Kontrollgruppe insbesondere Werte für Cholesterin, Estradiol ( $p<0,05)$ und Progesteron $(\mathrm{p}<0,01)$ erniedrigt.

Diskussion: Jede der analysierten pathologischen Entitäten wies ein spezifisches Lipid- und Steroid- 
lyzed entities displayed specific changes. However, since the changes were most obvious in pregnancies complicated by IUGR and only minor changes were observed in pregnancies where patients had impaired liver function, our data suggests that placental rather than maternal hepatic function strongly determines lipid and steroid levels in pregnancy.

\section{Introduction}

$\nabla$

Lipid metabolism and steroid hormone biosynthesis are intimately linked. The effects of estrogen on lipid and lipoprotein concentrations have been studied most extensively in postmenopausal hormone replacement therapy [1,2]. Orally administered estrogens increase triglyceride (TG) synthesis and triglyceriderich very-low-density lipoprotein cholesterol (VLDL) release from the liver [3]. The inhibiting effect of estrogen on hepatic lipase activity contributes to a reduction of TG catabolism [4]. Moreover, estrogen modulates low-density-lipoprotein (LDL)-receptor expression, thus regulating receptor-mediated LDL elimination [5]. During pregnancy, hepatic lipid metabolism is regulated by and interacts with steroid hormones released by the placenta, the primary endocrine organ producing pregnancy steroids. Placental estradiol is predominately synthesized from dehydroepiandrosterone (DHEA) sulfate precursors, provided by maternal and fetal adrenal glands at a ratio of approximately $40: 60$ [6]. Progesterone, however, is mainly produced from cholesterol and pregnenolone obtained from the maternal bloodstream. ApoB-containing LDL particles are considered to be the main source of cholesterol for placental progesterone synthesis [7]. - Fig. 1 shows a simplified interaction of lipids with the steroidogenic pathways of the materno-feto-placental unit.

As the pregnancy progresses, lipid and steroid hormone concentrations change markedly. After an initial reduction in the first eight weeks of pregnancy, plasma lipid concentrations increase steadily towards term. From the first to the third trimester, serum triglyceride (TG) concentrations rise by almost 300\%, which is at least partially attributable to the increase in circulating estrogen concentrations [8]. Total cholesterol (TC) and LDL levels rise by
hormon-Profil auf. Die deutlichsten Abweichungen von unkomplizierten Schwangerschaften zeigten Frauen mit IUGR. Schwangerschaften mit pathologischem Leberfunktionstest zeichneten sich durch eher geringe Veränderungen aus. Dies deutet darauf hin, dass Lipid- und Steroidspiegel während der Schwangerschaft stärker von der plazentaren als von der hepatischen Funktion bestimmt werden.

$25-50 \%$ and $70 \%$, respectively [9]. As LDL levels rise, there is a steady increase in progesterone concentrations [10].

Evidence is accumulating that the balance between lipid and steroid hormone levels is essential for maintaining a normal pregnancy. This is borne out by the fact that pathological pregnancies have been associated with altered lipid and steroid hormone profiles [11-16], a topic which is specifically addressed here later on. Given the important interactions between maternal liver and the placenta/fetoplacental unit for the regulation of lipids and steroid hormone metabolism, we hypothesized that pregnancy pathologies involving the placenta and/or the liver could affect pregnancy-specific lipid and steroid hormone interactions. In the present study, we first aimed to correlate lipid and steroid hormone concentrations in maternal serum in women with normal pregnancies through longitudinal measurements of serum levels of lipids and estradiol, progesterone, DHEA, and a further set of 9 characteristic progesterone or estrogen precursors and metabolites using highly specific gas chromatography and mass spectrometry (GC-MS) methods in healthy pregnant women. Secondly, we aimed to identify specific changes in these parameters in pregnancies complicated by intrauterine growth restriction (IUGR), preeclampsia (PE), HELLP syndrome, or intrahepatic cholestasis in pregnancy (ICP) compared to levels measured in uncomplicated pregnancies at the same gestational age.

\section{Material and Methods}

$\nabla$

The study was approved by the ethics committee of the Medical Faculty of the Rheinisch Westfaelische Technical University (RWTH) Aachen (EK 138/06, 119/08, 154/11). Written informed

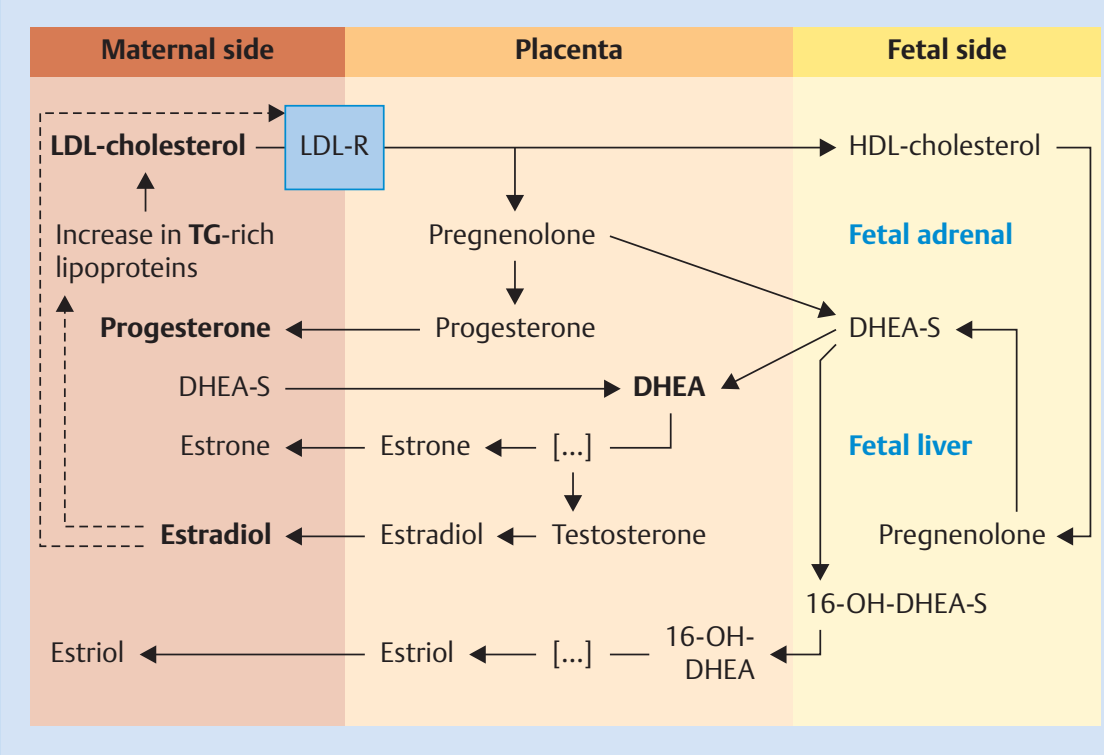

Fig. 1 Lipid and steroid hormone metabolism pathways in the materno-feto-placental unit (modified from Kallen et al. 2004). 
consent was obtained from each patient. From July 2008 to April 2010 , a total of 54 healthy normotensive pregnant women were prospectively included in this longitudinal study carried out in the Department of Obstetrics and Gynecology of the University Hospital of the RWTH Aachen (Study group A). Of those women, 21 were excluded because they were lost to follow-up, miscarried, developed pregnancy-related disease, had a preterm birth or refused further participation. In the remaining 33 patients, cubital vein blood was sampled and processed at four different time-points, with sampling done 3 times during pregnancy and once post partum as follows:

1. prior to gestational week 16 ,

2. between gestational week 22 and week 32 ,

3. after gestational week 34 , but before the onset of active labor,

4. more than four weeks post partum.

For further analysis patients were divided into smokers and nonsmokers, and obese (BMI $\geq 30$ ) and non-obese (according to the WHO classification). One woman who fulfilled both criteria (smoking and obesity) was subsequently excluded, yielding a total of 32 patients with five patients in the smoking group, five patients in the obesity group, and 22 patients in the control group (reference). The breast-feeding status post partum was recorded for 18 of the 22 patients of the reference group.

For the cross-sectional part of the study, 84 serum samples of patients at onset of disease and of patients with uncomplicated pregnancies (CTRL) matched for gestational age to the pathological pregnancy group and obtained between October 2006 and February 2012 were chosen from the biobank (Study group B). Gestational age was calculated based on the last menstrual period and verified by first trimester scan. Serum was sampled and processed as described; lipid values have been partly reported previously $[9,13]$.

Smokers and obese patients with BMI $\geq 30$ were excluded in the final analysis, yielding a total of 67 patients, with 13 patients in the PE group, 10 patients in the IUGR group, six patients with HELLP syndrome, seven with ICP, and 31 in the CTRL group.

\section{Definitions of study group B}

IUGR was defined in accordance with the ACOG guidelines and as described recently [13]. In brief, the following criteria had to be fulfilled:

estimated fetal weight $<10$ th percentile, plus

1. deceleration of fetal growth rate $>40$ th percentile on serial measurements, or

2. elevated resistance index on umbilical artery Doppler sonography above the 95th percentile, or absent or reversed end-diastolic blood flow, or

3. fetal asymmetry (head-to-abdominal circumference ratio $>$ 95th percentile), or

4. oligohydramnios (amniotic fluid index $<6 \mathrm{~cm}$ ).

Preeclampsia was defined in accordance with the ISSHP criteria and as described elsewhere [13] as follows: new onset of hypertension of more than $140 / 90 \mathrm{mmHg}$ and proteinuria of more than $300 \mathrm{mg} / \mathrm{d}$ or at least 2+ with urine dip-stick. Patients who had additional complications such as IUGR or HELLP syndrome were specifically excluded from the PE group.

HELLP syndrome was defined as recently described elsewhere $[17,18]$ and included upper abdominal pain together with confirmation of the following laboratory criteria in maternal serum analysis: hemolysis (haptoglobin levels below $30 \mathrm{mg} / \mathrm{dL}$ ); elevated liver enzyme activity (AST, ALT, LDH) three standard devia- tions above the mean; and low platelet count (below $100000 \mathrm{G}$ / L).

ICP was defined as a clinical finding of pruritus together with increased bile acid concentrations in maternal serum analysis (above $10 \mu \mathrm{mol} / \mathrm{L}$ ) and elevated liver enzyme activity (AST, ALT) above the reference values (> 35 U/L) [19] after HELLP syndrome was excluded.

General exclusion criteria for case and control groups were multiple gestation, fetal anomalies, abnormal fetal karyotype, patients with clinical or biochemical signs of infection, positive TORCH screening results, maternal diabetes mellitus or other severe maternal metabolic disorders, preterm birth following complications other than IUGR, PE, or HELLP syndrome, and the patient's withdrawal from the study. Blood samples were taken from women prior to or at least 5 days after administration of corticosteroids (betamethasone) to the mother for the induction of fetal lung maturity where clinically indicated. In line with previous publications [20] this did not result in significant differences in any of the tested parameters between patients who received betamethasone and those who did not (data not shown).

\section{Serum analysis}

\section{Lipid profile}

Blinded analysis of serum TG, TC, LDL-C and HDL-C was performed by colorimetric enzymatic methods using an automated photometric measuring unit (Roche/Hitachi Modular P800, Roche Diagnostics, Basel, Switzerland; triglycerides GPO-PAP reagent, cholesterol CHOD-PAP reagent, LDL-C plus 2nd generation reagent, HDL-C plus 3rd generation reagent, Cobas ${ }^{\circledR}$, Roche/Hitachi, Mannheim, Germany). Measurement ranges were: TG $=4$ $1000 \mathrm{mg} / \mathrm{dL}, \quad \mathrm{TC}=3-800 \mathrm{mg} / \mathrm{dL}, \quad$ LDL-C $=3-550 \mathrm{mg} / \mathrm{dL}$, and HDL-C $=3-120 \mathrm{mg} / \mathrm{dL}$.

\section{Steroid profile}

Steroid metabolites (DHEA, estradiol, and progesterone metabolites) were quantified by GC-MS in plasma (100-1000 $\mu \mathrm{l})$ using the following procedure [21]. Following an extraction step with $10 \mathrm{vol}$ dichloromethane, steroids were extracted in the organic phase containing unconjugated steroids and derivatized with $100 \mu l$ methoxyamine-HCl (Pierce Biotechnology, Rockford, IL, USA) in pyridine for $1 \mathrm{~h}$ at $60^{\circ} \mathrm{C}$. After evaporation under nitrogen, samples were incubated with $100 \mu$ l trimethylsilyl imidazole (Pierce Biotechnology, Rockford, IL, USA) for $16 \mathrm{~h}$ at $100^{\circ} \mathrm{C}$. The derivatives were purified by gel filtration on Lipidex-5000 columns (Perkin Elmer, Wellesley, MA, USA) and analyzed by GC-MS and selective ion monitoring (Agilent Technologies, Palo Alto, CA, USA). One characteristic ion was programmed for each steroid analyzed. A standard curve containing a mixture of known amounts of all steroids was performed for each extraction panel. Inter-assay variability was controlled using an aliquot of plasma pool and was below $10 \%$.

\section{Statistics}

Data analysis was carried out using the statistical package Prism Version 6.0e Software (GraphPad Software Inc., CA, USA). Clinical data are presented as means and 95\% confidence interval (95\% $\mathrm{CI}$ ) if metric. Categorical data are presented as percentages. Continuous variables are expressed as median $\pm 95 \% \mathrm{Cl}$ or as indicated in the respective figure. Correlations were analyzed by Spearman's correlation coefficient. Kruskal-Wallis test followed by Dunn's comparison post hoc test were done to compare measured variables between groups where indicated. Categorical da- 
Table 1 Demographic data of the study cohort of the longitudinal study group ( $A ; n=32$ ). Data are shown as means and $95 \%$ confidence intervals, or frequencies and percentages.

\begin{tabular}{|c|c|c|}
\hline \multirow[t]{3}{*}{ Maternal age (years) } & mean & 31.0 \\
\hline & lower $95 \% \mathrm{Cl}$ of mean & 29.0 \\
\hline & upper $95 \% \mathrm{Cl}$ of mean & 33.0 \\
\hline \multirow[t]{3}{*}{ Maternal pre-pregnancy BMI $\left(\mathrm{kg} / \mathrm{m}^{2}\right)$} & mean & 23.8 \\
\hline & lower $95 \% \mathrm{Cl}$ of mean & 21.8 \\
\hline & upper $95 \% \mathrm{Cl}$ of mean & 25.7 \\
\hline \multirow[t]{2}{*}{ Maternal overweight/obesity } & $\%$ & 15.6 \\
\hline & $\mathrm{n}$ & 5 \\
\hline \multirow{2}{*}{$\begin{array}{l}\text { Assisted reproductive technologies } \\
\text { (ART) }\end{array}$} & $\%$ & 0 \\
\hline & n & 0 \\
\hline \multirow[t]{2}{*}{ Primiparity } & $\%$ & 84.4 \\
\hline & $n$ & 27 \\
\hline \multirow[t]{2}{*}{ Actual smoking status } & $\%$ & 15.6 \\
\hline & $n$ & 5 \\
\hline \multirow[t]{3}{*}{ Weeks of gestation at delivery } & mean & 40.2 \\
\hline & lower $95 \% \mathrm{Cl}$ of mean & 39.7 \\
\hline & upper $95 \% \mathrm{Cl}$ of mean & 40.6 \\
\hline \multirow[t]{2}{*}{ Mode of delivery (c-section) } & $\%$ & 25.0 \\
\hline & $n$ & 8 \\
\hline \multirow[t]{2}{*}{ Neonatal gender (female) } & $\%$ & 53.1 \\
\hline & $n$ & 17 \\
\hline \multirow[t]{3}{*}{ Neonatal birth weight $(\mathrm{g})$} & mean & 3531 \\
\hline & lower $95 \% \mathrm{Cl}$ of mean & 3370 \\
\hline & upper $95 \% \mathrm{Cl}$ of mean & 3692 \\
\hline \multirow[t]{3}{*}{ Neonatal birth weight, percentiles } & mean & 50.2 \\
\hline & lower $95 \% \mathrm{Cl}$ of mean & 41.2 \\
\hline & upper $95 \% \mathrm{Cl}$ of mean & 59.2 \\
\hline \multirow{3}{*}{$\begin{array}{l}\text { Weeks of gestation at 1st blood } \\
\text { collection (weeks) }\end{array}$} & mean & 10.0 \\
\hline & lower $95 \% \mathrm{Cl}$ of mean & 9.0 \\
\hline & upper $95 \% \mathrm{Cl}$ of mean & 11.1 \\
\hline \multirow{3}{*}{$\begin{array}{l}\text { Weeks of gestation at 2nd blood } \\
\text { collection (weeks) }\end{array}$} & mean & 26.2 \\
\hline & lower $95 \% \mathrm{Cl}$ of mean & 25.3 \\
\hline & upper $95 \% \mathrm{Cl}$ of mean & 27.1 \\
\hline \multirow{3}{*}{$\begin{array}{l}\text { Weeks of gestation at 3rd blood } \\
\text { collection (weeks) }\end{array}$} & mean & 36.5 \\
\hline & lower $95 \% \mathrm{Cl}$ of mean & 36.0 \\
\hline & upper $95 \% \mathrm{Cl}$ of mean & 37.0 \\
\hline \multirow{3}{*}{$\begin{array}{l}\text { Weeks post partum at blood } \\
\text { collection (weeks) }\end{array}$} & mean & 12.8 \\
\hline & lower $95 \% \mathrm{Cl}$ of mean & 4.4 \\
\hline & upper $95 \% \mathrm{Cl}$ of mean & 21.1 \\
\hline
\end{tabular}

ta were analyzed by $\mathrm{X}^{2}$ test. Values of $\mathrm{p}<0.05$ were regarded as significant.

\section{Results}

$\nabla$

\section{Group A - longitudinal study group}

The clinical characteristics of the longitudinal study group are presented in 0 Table 1. Mean gestational age at blood sampling was the 11 th, 27th, and 37 th week of gestation, and 13 weeks post partum.

\section{Lipid profile of study group A}

Lipid concentrations over the course of pregnancy in non-smoking and non-obese patients are presented in 0 Table 2. Mean TG levels increased by $175 \%$ from the first to the third trimester, fol- lowed by a drop post partum to early pregnancy values. TC levels increased by $60 \%$ and dropped post partum, but were still $27 \%$ higher than in early pregnancy.

The increase in TC levels was attributed mainly to increasing LDL levels (85\%). LDL levels remained higher post partum compared to levels in early pregnancy. After an initial rise in HDL concentration by $28 \%$ from the first to the second sampling time-point, HDL levels dropped steadily to first trimester values post partum (- Table 2).

In the 13 breast-feeding patients HDL levels were significantly higher compared to the five non-breast-feeding patients (median $68 \mathrm{mg} / \mathrm{dL}$ [95\% CI 58-75] vs. 52 [41-74], $\mathrm{p}=0.0247$ ). This resulted in a significantly lower LDL/HDL ratio in the breast-feeding group (median 1.76 [95\% CI 1.13-2.2] vs. 2.62 [1.55-4.4], p=0.0460). There were no significant differences in the other lipid values between breast-feeding and non-breast-feeding patients (data not shown). Neither smoking nor obesity significantly altered changes in TG and TC levels (data not shown). LDL was not affected by smoking status or BMI (data not shown), HDL levels were significantly lower in obese patients in the first trimester (median $47 \mathrm{mg} / \mathrm{dL}$ [95\% CI 40.5-51.9] vs. 61 [57.2-68.6], $\mathrm{p}=0.0022$ ) and post partum (median $52 \mathrm{mg} / \mathrm{dL}$ [95\% CI] vs. 67 [61.2-63.5], $\mathrm{p}=0.0035$ ) as compared to non-obese patients.

\section{Steroid profile of study group A}

As expected, progesterone and estradiol levels reached their maximum levels after 34 weeks of gestation while DHEA dropped to its minimum level in the same time period ( $\triangle$ Table 2). No differences were observed for fetal gender (data not shown). Smoking resulted in significantly reduced estradiol concentrations in the third trimester compared to the non-smoking reference group (median $22.9 \mathrm{ng} / \mathrm{mL}$ [95\% CI 16.5-27.0] vs. 28.3 [95\% CI 24.7-30-6], $\mathrm{p}=0.0239$ ). Post partum, the steroid hormone concentrations did not differ from first trimester values, independently of whether or not the patients were still breastfeeding (data not shown).

The extended metabolome profile differed significantly over the course of pregnancy in three of nine analytes: $3 \alpha, 5 \alpha$-tetrahydroprogesterone allopregnanolone, $20 \alpha$-dihydro-3 $\beta, 5 \alpha$-tetrahydroprogesterone, and estriol. Those three metabolites increased as pregnancy progressed and were significantly higher in the third trimester compared to the first trimester and dropped post partum to the levels observed in the first trimester or lower ( Table 2). Correlation analysis was done to quantify the association between lipid values and the primary steroid hormone targets. Lipid values were positively correlated to estradiol (TG $\rho=0.66$, $\mathrm{p}<0.0001$; TC $\rho=0.53, \mathrm{p}<0.0001 ;$ LDL $\rho=0.42, \mathrm{p}<0.0001 ; \mathrm{HDL}$ $\rho=0.21, p=0.051$ ) and progesterone concentrations (TG $\rho=0.57$, $\mathrm{p}<0.0001$; TC $\rho=0.45, \mathrm{p}<0.0001$; LDL $\rho=0.35, \mathrm{p}<0.001$; HDL $\rho=0.24, p=0.024$ ) and negatively correlated to DHEA levels (TG $\rho=-0.31, p=0.003 ;$ TC $\rho=-0.37, p<0.0001 ;$ LDL $\rho=-0.33$, $\mathrm{p}=0.002 ; \operatorname{HDL} \rho=-0.21, \mathrm{p}=0.053)$.

\section{Group B - cross-sectional study group}

with pathological pregnancies

The clinical characteristics of the cross-sectional study groups are presented in Table 3. Outcome data are missing for one patient in the ICP group because she was lost to follow-up. Following the exclusion of smokers and obese patients, mean maternal age, BMI, and weeks of gestation at blood collection did not differ significantly between the study groups compared to the CTRL group 
Table 2 Lipid profiles and progesterone and estrogen metabolome in the longitudinal study arm ( $;$; $=22$ after exclusion of obese patients and smokers). Data are presented as median and $95 \%$ confidence intervals. Significant p-values for the comparison of time-points during pregnancy and post partum versus first trimester are indicated as follows: ${ }^{*}<0.05,{ }^{* *}<0.01,{ }^{* *}<0.001,{ }^{* * *}<0.0001$. a) WoG $22-32$ vs. WoG $<16$, b) WoG $>34$ vs. WoG $<16$, c) p.p. vs. WoG $<16$.

\begin{tabular}{|c|c|c|c|c|c|c|c|c|}
\hline Name & Chemical name & Units & & $\begin{array}{l}\text { WoG } \\
<16\end{array}$ & $\begin{array}{l}\text { WoG } \\
22-32 \text { a) }\end{array}$ & $\begin{array}{l}\text { WoG } \\
>34 \text { b) }\end{array}$ & p.p. c) & p-value \\
\hline \multicolumn{9}{|l|}{ Lipid profile } \\
\hline \multirow[t]{3}{*}{ LDL cholesterol } & & $\mathrm{mg} / \mathrm{dL}$ & median & 80.5 & 129.5 & 146.5 & 128.5 & a) $)^{* * * *}$ \\
\hline & & & lower $95 \% \mathrm{Cl}$ & 71.0 & 106.0 & 117.0 & 89.0 & b) ${ }^{* * * *}$ \\
\hline & & & upper $95 \% \mathrm{Cl}$ & 85.0 & 160.0 & 199.0 & 139.0 & c) $)^{* *}$ \\
\hline \multirow[t]{3}{*}{ HDL cholesterol } & & $\mathrm{mg} / \mathrm{dL}$ & median & 61.0 & 80.5 & 75.5 & 67.0 & a) ${ }^{* *}$ \\
\hline & & & lower $95 \% \mathrm{Cl}$ & 55.0 & 67.0 & 58.0 & 56.0 & \\
\hline & & & upper $95 \% \mathrm{Cl}$ & 71.0 & 94.0 & 90.0 & 75.0 & \\
\hline \multirow[t]{3}{*}{ Total cholesterol } & & $\mathrm{mg} / \mathrm{dL}$ & median & 160.5 & 235.0 & 264.5 & 212.5 & $a)^{* * *}$ \\
\hline & & & lower $95 \% \mathrm{Cl}$ & 152.0 & 214.0 & 236.0 & 178.0 & b) $)^{* * * *}$ \\
\hline & & & upper $95 \% \mathrm{Cl}$ & 181.0 & 258.0 & 291.0 & 238.0 & \\
\hline \multirow[t]{3}{*}{ Triglycerides } & & $\mathrm{mg} / \mathrm{dL}$ & median & 88.5 & 165.0 & 241.0 & 95.0 & a) $)^{* * * *}$ \\
\hline & & & lower $95 \% \mathrm{Cl}$ & 66.0 & 108.0 & 190.0 & 66.0 & b) $)^{* * * *}$ \\
\hline & & & upper $95 \% \mathrm{Cl}$ & 102.0 & 245.0 & 296.0 & 128.0 & c) $)^{* *}$ \\
\hline \multicolumn{9}{|l|}{ Basic steroid hormone profile } \\
\hline \multirow[t]{3}{*}{ Progesterone } & pregn-4-ene-3,20-dione & $\mathrm{ng} / \mathrm{mL}$ & median & 48.3 & 75.2 & 113.5 & 23.2 & a) $)^{* *}$ \\
\hline & & & lower $95 \% \mathrm{Cl}$ & 33.1 & 58.5 & 96.7 & 18.6 & b) ${ }^{* * * *}$ \\
\hline & & & upper $95 \% \mathrm{Cl}$ & 59.9 & 103.2 & 135.6 & 35.7 & \\
\hline \multirow[t]{3}{*}{ Estradiol (E2) } & 17ß-estradiol & $\mathrm{ng} / \mathrm{mL}$ & median & 14.0 & 19.7 & 28.3 & 13.3 & a) ${ }^{* * *}$ \\
\hline & & & lower 95\% Cl & 12.9 & 18.8 & 24.7 & 12.5 & b) $)^{* * * *}$ \\
\hline & & & upper $95 \% \mathrm{Cl}$ & 15.4 & 23.8 & 30.6 & 15.4 & \\
\hline \multirow[t]{3}{*}{ DHEA } & 5 -androsten-3 $\beta$-ol-17-one & $\mathrm{ng} / \mathrm{mL}$ & median & 27.3 & 19.3 & 18.9 & 29.6 & a) ${ }^{*}$ \\
\hline & & & lower $95 \% \mathrm{Cl}$ & 22.2 & 12.9 & 10.7 & 24.8 & b) ${ }^{* *}$ \\
\hline & & & upper $95 \% \mathrm{Cl}$ & 38.4 & 24.6 & 25.5 & 34.1 & \\
\hline \multicolumn{9}{|l|}{ Extended metabolome profile } \\
\hline \multirow[t]{3}{*}{$6 \beta$-hydroxy-progesterone } & 4-pregnen-6 6 -ol-3,20-dione & $\mathrm{ng} / \mathrm{mL}$ & median & 7.9 & 8.3 & 8.8 & 9.3 & \\
\hline & & & lower $95 \% \mathrm{Cl}$ & 7.2 & 7.1 & 7.5 & 7.8 & \\
\hline & & & upper $95 \% \mathrm{Cl}$ & 10.7 & 10.5 & 9.6 & 10.2 & \\
\hline \multirow[t]{3}{*}{$5 \beta$-dihydro-progesterone } & 5ß-pregnan-3,20-dione & $\mathrm{ng} / \mathrm{mL}$ & median & 32.4 & 32.3 & 33.6 & 31.6 & \\
\hline & & & lower 95\% Cl & 27.6 & 29.7 & 28.9 & 27.5 & \\
\hline & & & upper $95 \% \mathrm{Cl}$ & 48.2 & 36.3 & 37.4 & 36.8 & \\
\hline \multirow[t]{3}{*}{$5 \alpha$-dihydro-progesterone } & 5 $\alpha$-pregnan-3,20-dione & $\mathrm{ng} / \mathrm{mL}$ & median & 118.3 & 114.4 & 113.7 & 115.6 & \\
\hline & & & lower $95 \% \mathrm{Cl}$ & 96.2 & 91.5 & 93.0 & 91.5 & \\
\hline & & & upper $95 \% \mathrm{Cl}$ & 131.4 & 135.7 & 127.5 & 139.9 & \\
\hline \multirow{3}{*}{$\begin{array}{l}3 \alpha, 5 \alpha \text {-tetrahydro-progester- } \\
\text { one allopregnanolone }\end{array}$} & 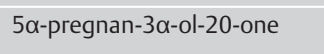 & $\mathrm{ng} / \mathrm{mL}$ & median & 6.4 & 8.5 & 12.0 & 5.0 & b) ${ }^{* * * *}$ \\
\hline & & & lower $95 \% \mathrm{Cl}$ & 5.1 & 7.2 & 10.5 & 4.2 & \\
\hline & & & upper $95 \% \mathrm{Cl}$ & 8.0 & 11.4 & 13.8 & 6.1 & \\
\hline \multirow{3}{*}{$\begin{array}{l}20 \alpha \text {-dihydro- } 3 \beta, 5 \alpha \text {-tetrahydro- } \\
\text { progesterone }\end{array}$} & 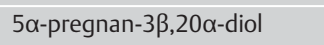 & $\mathrm{ng} / \mathrm{mL}$ & median & 2.6 & 3.5 & 4.6 & 1.2 & a) ${ }^{*}$ \\
\hline & & & lower $95 \% \mathrm{Cl}$ & 2.0 & 3.0 & 3.9 & 0.9 & b) ${ }^{* * *}$ \\
\hline & & & upper $95 \% \mathrm{Cl}$ & 2.8 & 4.0 & 6.2 & 1.4 & $c)^{*}$ \\
\hline \multirow[t]{3}{*}{ 17-hydroxy-progesterone } & 4-pregnen-17 $\alpha$-ol-3,20-dione & $\mathrm{ng} / \mathrm{mL}$ & median & 23.8 & 24.0 & 23.5 & 23.4 & \\
\hline & & & lower $95 \% \mathrm{Cl}$ & 21.8 & 22.5 & 22.1 & 21.7 & \\
\hline & & & upper $95 \% \mathrm{Cl}$ & 24.7 & 25.4 & 24.4 & 26.9 & \\
\hline \multirow[t]{3}{*}{$17 \alpha, 20 \alpha$-dihydro-progesterone } & 4-pregnen-17,20 $\alpha$-diol-3-one & $\mathrm{ng} / \mathrm{mL}$ & median & 18.2 & 17.0 & 16.0 & 17.1 & \\
\hline & & & lower $95 \% \mathrm{Cl}$ & 13.8 & 12.5 & 12.7 & 14.9 & \\
\hline & & & upper $95 \% \mathrm{Cl}$ & 21.6 & 20.8 & 17.9 & 19.1 & \\
\hline \multirow[t]{3}{*}{ 11-keto-progesterone } & 4-pregnen-3,11,20-trione & $\mathrm{ng} / \mathrm{mL}$ & median & 65.0 & 67.5 & 63.3 & 63.6 & \\
\hline & & & lower $95 \% \mathrm{Cl}$ & 57.2 & 65.4 & 59.7 & 56.5 & \\
\hline & & & upper $95 \% \mathrm{Cl}$ & 67.8 & 75.8 & 67.6 & 67.6 & \\
\hline \multirow[t]{3}{*}{ Estriol (E3) } & $\begin{array}{l}\text { 1,3,5(10)-estratrien- } \\
3,16 \alpha, 17 \beta \text {-triol }\end{array}$ & $\mathrm{ng} / \mathrm{mL}$ & median & 8.3 & 11.5 & 16.0 & 8.8 & a) ${ }^{* * *}$ \\
\hline & & & lower $95 \% \mathrm{Cl}$ & 8.0 & 10.7 & 13.5 & 8.0 & b) $)^{* * * *}$ \\
\hline & & & upper $95 \% \mathrm{Cl}$ & 9.8 & 12.4 & 18.5 & 9.6 & \\
\hline
\end{tabular}

WoG $=$ week of gestation, p.p. $=$ post partum

as indicated by the non-overlapping of the $95 \%$ confidence intervals.

Blood pressure was, by definition, elevated in the PE and HELLP groups. Compared to the CTRL group, patients in these groups were delivered earlier, and their neonates had lower birth weights. Although fetal gender was balanced in the CTRL, PE, and IUGR groups, female neonates were more common in the HELLP and ICP groups. 
Table 3 Demographic data and clinical data of the study cohort of the cross-sectional study group (B; $\mathrm{n}=67)$. Data are presented as means and $95 \%$ confidence intervals, or frequencies and percentages. A p-value $<0.05$ indicated group significance.

\begin{tabular}{|c|c|c|c|c|c|c|c|}
\hline & & CTRL & PE & IUGR & HELLP & ICP & p-value \\
\hline & & $\mathbf{n}=\mathbf{3 1}$ & $n=13$ & $n=10$ & $n=6$ & $n=7$ & \\
\hline \multirow{3}{*}{ Maternal age (years) } & Mean & 31.25 & 31.22 & 30.65 & 37.12 & 31.74 & 0.2739 \\
\hline & lower $95 \% \mathrm{Cl}$ of mean & 29.16 & 27.73 & 26.52 & 31.39 & 25.83 & \\
\hline & upper $95 \% \mathrm{Cl}$ of mean & 33.35 & 34.72 & 34.78 & 42.84 & 37.65 & \\
\hline \multirow[t]{3}{*}{ Maternal pre-pregnancy BMI $\left(\mathrm{kg} / \mathrm{m}^{2}\right)$} & Mean & 22.72 & 25.18 & 22.03 & 22.76 & 23.59 & 0.1925 \\
\hline & lower $95 \% \mathrm{Cl}$ of mean & 21.6 & 23.18 & 19.58 & 18.99 & 19.83 & \\
\hline & upper $95 \% \mathrm{Cl}$ of mean & 23.83 & 27.17 & 24.48 & 26.53 & 27.34 & \\
\hline \multirow[t]{2}{*}{ Assisted reproductive technologies (ART) } & $\%$ & 3.2 & 7.7 & 10.0 & 0.0 & 14.2 & 0.7326 \\
\hline & $\mathrm{n}$ & 1 & 1 & 1 & 0 & 1 & \\
\hline \multirow[t]{2}{*}{ Primiparity } & $\%$ & 54.84 & 53.85 & 70.00 & 66.67 & 71.43 & 0.8351 \\
\hline & $\mathrm{n}$ & 17 & 7 & 7 & 4 & 3 & \\
\hline \multirow{3}{*}{ Systolic blood pressure $(\mathrm{mmHg})$} & mean & 114.8 & 149.8 & 121.2 & 156.5 & 120.1 & $<0.0001$ \\
\hline & lower $95 \% \mathrm{Cl}$ of mean & 110.4 & 144.5 & 106 & 131.4 & 109.4 & \\
\hline & upper $95 \% \mathrm{Cl}$ of mean & 119.3 & 155.2 & 136.4 & 181.6 & 130.9 & \\
\hline \multirow[t]{3}{*}{ Diastolic blood pressure $(\mathrm{mmHg})$} & mean & 68 & 91.38 & 69.6 & 91.5 & 71 & $<0.0001$ \\
\hline & lower $95 \% \mathrm{Cl}$ of mean & 64.63 & 86.23 & 60.14 & 82.14 & 64.08 & \\
\hline & upper $95 \% \mathrm{Cl}$ of mean & 71.37 & 96.54 & 79.06 & 100.9 & 77.92 & \\
\hline \multirow[t]{3}{*}{ Weeks of gestation at sampling } & mean & 33.27 & 33.87 & 32.17 & 32.38 & 35.06 & 0.8420 \\
\hline & lower $95 \% \mathrm{Cl}$ of mean & 31.4 & 30.8 & 27.46 & 27.53 & 31.59 & \\
\hline & upper $95 \% \mathrm{Cl}$ of mean & 35.14 & 36.94 & 36.89 & 37.23 & 38.54 & \\
\hline \multirow[t]{3}{*}{ Weeks of gestation at delivery } & mean & 39.28 & 34.68 & 32.44 & 32.38 & 37.67 & $<0.0001$ \\
\hline & lower $95 \% \mathrm{Cl}$ of mean & 38.84 & 31.78 & 27.81 & 27.53 & 36.55 & \\
\hline & upper $95 \% \mathrm{Cl}$ of mean & 39.71 & 37.58 & 37.08 & 37.23 & 38.79 & \\
\hline \multirow[t]{2}{*}{ Mode of delivery (c-section) } & $\%$ & 45.16 & 84.62 & 90.00 & 100.00 & 42.86 & 0.0050 \\
\hline & $\mathrm{n}$ & 14 & 11 & 9 & 6 & 3 & \\
\hline \multirow[t]{2}{*}{ Neonatal gender (female) } & $\%$ & 54.8 & 38.5 & 50.0 & 83.3 & 100.0 & 0.0589 \\
\hline & $\mathrm{N}$ & 17 & 5 & 5 & 5 & 7 & \\
\hline \multirow[t]{3}{*}{ Neonatal birth weight (g) } & mean & 3424 & 2242 & 1344 & 1818 & 3123 & $<0.0001$ \\
\hline & lower $95 \% \mathrm{Cl}$ of mean & 3289 & 1649 & 650.8 & 908.9 & 2715 & \\
\hline & upper $95 \% \mathrm{Cl}$ of mean & 3559 & 2834 & 2036 & 2726 & 3530 & \\
\hline \multirow[t]{3}{*}{ Neonatal birth weight percentiles } & mean & 52.3 & 29.3 & 2.1 & 31.6 & 49.0 & $<0.0001$ \\
\hline & lower $95 \% \mathrm{Cl}$ of mean & 43.6 & 16.2 & 1.0 & 5.7 & 19.1 & \\
\hline & upper $95 \% \mathrm{Cl}$ of mean & 61.0 & 42.3 & 3.2 & 57.5 & 78.9 & \\
\hline \multirow[t]{3}{*}{ Proteinuria (mg/24 h) } & mean & $\mathrm{N} / \mathrm{A}$ & 2964 & $\mathrm{~N} / \mathrm{A}$ & $\mathrm{N} / \mathrm{A}$ & N/A & \\
\hline & lower $95 \% \mathrm{Cl}$ of mean & $\mathrm{N} / \mathrm{A}$ & 357 & $\mathrm{~N} / \mathrm{A}$ & $\mathrm{N} / \mathrm{A}$ & $\mathrm{N} / \mathrm{A}$ & \\
\hline & upper $95 \% \mathrm{Cl}$ of mean & $\mathrm{N} / \mathrm{A}$ & 5572 & N/A & N/A & $\mathrm{N} / \mathrm{A}$ & \\
\hline \multirow[t]{3}{*}{ AST (U/L) } & mean & 23.0 & 27.4 & 22.5 & 437.3 & 172.7 & $<0.0001$ \\
\hline & lower $95 \% \mathrm{Cl}$ of mean & 20.6 & 22.4 & 20.0 & 156.5 & 83.4 & \\
\hline & upper $95 \% \mathrm{Cl}$ of mean & 25.3 & 32.4 & 25.0 & 718.2 & 262.1 & \\
\hline \multirow[t]{3}{*}{$\operatorname{ALT}(\mathrm{U} / \mathrm{L})$} & mean & 13.7 & 18.7 & 13.9 & 402.8 & 283.6 & $<0.0001$ \\
\hline & lower $95 \% \mathrm{Cl}$ of mean & 11.8 & 13.1 & 10.6 & 194.9 & 115.9 & \\
\hline & upper $95 \% \mathrm{Cl}$ of mean & 15.5 & 24.3 & 17.2 & 610.7 & 451.2 & \\
\hline \multirow[t]{3}{*}{ Platelets (G/L) } & mean & 264.9 & 217.3 & 238.5 & 45.8 & 267.6 & 0.0002 \\
\hline & lower $95 \% \mathrm{Cl}$ of mean & 242.8 & 175.7 & 200.9 & 18.4 & 156.5 & \\
\hline & upper $95 \% \mathrm{Cl}$ of mean & 287.0 & 258.9 & 276.1 & 73.2 & 378.7 & \\
\hline \multirow[t]{3}{*}{ Haptoglobin $(\mathrm{mg} / \mathrm{mL})$} & mean & $\mathrm{N} / \mathrm{A}$ & $\mathrm{N} / \mathrm{A}$ & $\mathrm{N} / \mathrm{A}$ & 8.4 & 101.0 & \\
\hline & lower $95 \% \mathrm{Cl}$ of mean & $\mathrm{N} / \mathrm{A}$ & N/A & $\mathrm{N} / \mathrm{A}$ & -2.5 & 48.9 & \\
\hline & upper $95 \% \mathrm{Cl}$ of mean & N/A & N/A & $\mathrm{N} / \mathrm{A}$ & 19.3 & 153.1 & \\
\hline \multirow[t]{3}{*}{ Bile acids ( $\mu \mathrm{mol} / \mathrm{L})$} & mean & $\mathrm{N} / \mathrm{A}$ & $\mathrm{N} / \mathrm{A}$ & $\mathrm{N} / \mathrm{A}$ & $\mathrm{N} / \mathrm{A}$ & 30.17 & \\
\hline & lower $95 \% \mathrm{Cl}$ of mean & $\mathrm{N} / \mathrm{A}$ & $\mathrm{N} / \mathrm{A}$ & $\mathrm{N} / \mathrm{A}$ & N/A & 20.61 & \\
\hline & upper $95 \% \mathrm{Cl}$ of mean & $\mathrm{N} / \mathrm{A}$ & $\mathrm{N} / \mathrm{A}$ & N/A & $\mathrm{N} / \mathrm{A}$ & 39.73 & \\
\hline
\end{tabular}

Lipid profile of study group B

Lipid profiles of the different groups are summarized in $\odot$ Table 4. Compared to the CTRL group, LDL concentrations were significantly lower in the IUGR group. TC levels were significantly lower in the IUGR and the HELLP groups. HDL levels were lower in the ICP group.

\section{Steroid profile of study group B}

Progesterone and estradiol concentrations were lower in the IUGR group compared to the CTRL group, but otherwise, no other significant differences were observed ( $\bigcirc$ Table 4$)$. In the extended metabolome profile, significant group differences were found for three of nine steroid hormones ( $3 \alpha, 5 \alpha$-tetrahydro-progesterone allopregnanolone, 11-keto-progesterone, and estriol) as indicated in $\odot$ Table 4. 
Table 4 Lipid profiles and progesterone and estrogen metabolome in the cross-sectional study arm (B; $n=67)$. Data are presented as median and $95 \%$ confidence intervals. Significant differences in concentrations between the respective study group vs. the CTRL group are indicated as follows: ${ }^{*} p<0.05,{ }^{* *} p<0.01$, ${ }^{* * *} \mathrm{p}<0.001,{ }^{* * * *} \mathrm{p}<0.0001$. a) PE vs. CTRL, b) IUGR vs. CTRL, c) HELLP vs. CTRL, d) ICP vs. CTRL.

\begin{tabular}{|c|c|c|c|c|c|c|c|c|c|}
\hline Name & Chemical name & Units & & CTRL & PE a) & IUGR b) & HELLP c) & ICP d) & p-value \\
\hline \multicolumn{10}{|l|}{ Lipid profile } \\
\hline \multirow[t]{3}{*}{ LDL cholesterol } & & $\mathrm{mg} / \mathrm{dL}$ & median & 136 & 152 & 103.5 & 109.5 & 188 & b) ${ }^{*}$ \\
\hline & & & lower $95 \% \mathrm{Cl}$ & 128 & 93 & 67 & 61 & 141 & \\
\hline & & & upper $95 \% \mathrm{Cl}$ & 162 & 193 & 141 & 148 & 335 & \\
\hline \multirow[t]{3}{*}{ HDL cholesterol } & & $\mathrm{mg} / \mathrm{dL}$ & median & 79 & 64 & 81 & 68 & 57 & d) $* *$ \\
\hline & & & lower $95 \% \mathrm{Cl}$ & 71 & 45 & 56 & 40 & 48 & \\
\hline & & & upper $95 \% \mathrm{Cl}$ & 84 & 86 & 107 & 128 & 62 & \\
\hline \multirow[t]{3}{*}{ Total cholesterol } & & $\mathrm{mg} / \mathrm{dL}$ & median & 264 & 260 & 231 & 206.5 & 299 & a) ${ }^{*}$ \\
\hline & & & lower $95 \% \mathrm{Cl}$ & 247 & 203 & 188 & 182 & 221 & b) ${ }^{*}$ \\
\hline & & & upper $95 \% \mathrm{Cl}$ & 276 & 331 & 251 & 249 & 454 & \\
\hline \multirow[t]{3}{*}{ Triglycerides } & & $\mathrm{mg} / \mathrm{dL}$ & median & 219 & 304 & 174 & 247 & 261 & \\
\hline & & & lower $95 \% \mathrm{Cl}$ & 168 & 178 & 121 & 88 & 170 & \\
\hline & & & upper $95 \% \mathrm{Cl}$ & 267 & 444 & 256 & 643 & 345 & \\
\hline \multicolumn{10}{|c|}{ Basic steroid hormone profile } \\
\hline \multirow[t]{3}{*}{ Progesterone } & pregn-4-ene-3,20-dione & $\mathrm{ng} / \mathrm{mL}$ & median & 98.1 & 98.16 & 51.32 & 110.6 & 105.7 & b) $)^{* *}$ \\
\hline & & & lower $95 \% \mathrm{Cl}$ & 75.58 & 81.74 & 37.99 & 43.22 & 72.37 & \\
\hline & & & upper $95 \% \mathrm{Cl}$ & 109.9 & 125.4 & 78.65 & 180.3 & 134.8 & \\
\hline \multirow[t]{3}{*}{ Estradiol (E2) } & 17ß-estradiol & $\mathrm{ng} / \mathrm{mL}$ & median & 21.57 & 21.4 & 17.59 & 21.59 & 31.36 & b) ${ }^{*}$ \\
\hline & & & lower $95 \% \mathrm{Cl}$ & 19.12 & 16.77 & 12.48 & 10.43 & 21.28 & \\
\hline & & & upper $95 \% \mathrm{Cl}$ & 27.85 & 24.86 & 22.08 & 28.04 & 34.26 & \\
\hline \multirow[t]{3}{*}{ DHEA } & 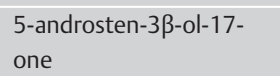 & $\mathrm{ng} / \mathrm{mL}$ & median & 20.24 & 24.47 & 22.69 & 17.95 & 17.56 & \\
\hline & & & lower $95 \% \mathrm{Cl}$ & 13.72 & 17.11 & 6.12 & 11.54 & 11.49 & \\
\hline & & & upper $95 \% \mathrm{Cl}$ & 24.3 & 41.75 & 38.19 & 28.96 & 24.53 & \\
\hline \multicolumn{10}{|c|}{ Extended metabolome profile } \\
\hline \multirow[t]{3}{*}{$6 \beta$-hydroxy-progesterone } & 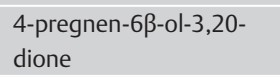 & $\mathrm{ng} / \mathrm{mL}$ & median & 9.1 & 9.9 & 9.8 & 10.0 & 10.4 & \\
\hline & & & lower $95 \% \mathrm{Cl}$ & 8.1 & 7.0 & 8.6 & 7.9 & 8.2 & \\
\hline & & & upper $95 \% \mathrm{Cl}$ & 10.4 & 15.0 & 11.8 & 18.4 & 11.6 & \\
\hline \multirow[t]{3}{*}{$5 \beta$-dihydro-progesterone } & $5 \beta$-pregnan-3,20-dione & $\mathrm{ng} / \mathrm{mL}$ & median & 16.2 & 19.1 & 17.5 & 17.3 & 15.5 & \\
\hline & & & lower $95 \% \mathrm{Cl}$ & 14.2 & 15.6 & 12.7 & 10.6 & 11.8 & \\
\hline & & & upper $95 \% \mathrm{Cl}$ & 18.7 & 27.2 & 23.3 & 23.5 & 24.4 & \\
\hline \multirow[t]{3}{*}{$5 \alpha$-dihydro-progesterone } & $5 \alpha$-pregnan-3,20-dione & $\mathrm{ng} / \mathrm{mL}$ & median & 105.9 & 129.1 & 84.8 & 137.7 & 114.3 & \\
\hline & & & lower $95 \% \mathrm{Cl}$ & 93.7 & 121.1 & 58.2 & 86.4 & 47.7 & \\
\hline & & & upper $95 \% \mathrm{Cl}$ & 133.2 & 180.4 & 151.7 & 730.0 & 173.4 & \\
\hline \multirow[t]{3}{*}{$\begin{array}{l}3 \alpha, 5 \alpha \text {-tetrahydro-proges- } \\
\text { terone allopregnanolone }\end{array}$} & 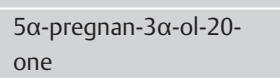 & $\mathrm{ng} / \mathrm{mL}$ & median & 15.7 & 13.6 & 8.1 & 16.2 & 13.9 & b) $)^{* *}$ \\
\hline & & & lower $95 \% \mathrm{Cl}$ & 11.6 & 11.3 & 6.6 & 8.8 & 9.5 & \\
\hline & & & upper $95 \% \mathrm{Cl}$ & 16.6 & 15.4 & 13.7 & 32.5 & 21.6 & \\
\hline \multirow{3}{*}{$\begin{array}{l}20 \alpha \text {-dihydro-3 } \beta, 5 \alpha \text {-tetra- } \\
\text { hydro-progesterone }\end{array}$} & 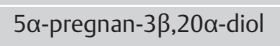 & $\mathrm{ng} / \mathrm{mL}$ & median & 6.9 & 7.4 & 5.6 & 7.9 & 8.2 & \\
\hline & & & lower $95 \% \mathrm{Cl}$ & 6.0 & 5.6 & 2.4 & 4.8 & 6.1 & \\
\hline & & & upper $95 \% \mathrm{Cl}$ & 7.5 & 11.2 & 8.4 & 18.9 & 16.6 & \\
\hline \multirow[t]{3}{*}{ 17-hydroxy-progesterone } & $\begin{array}{l}\text { 4-pregnen-17 } \alpha-o l-3,20- \\
\text { dione }\end{array}$ & $\mathrm{ng} / \mathrm{mL}$ & median & 21.1 & 24.6 & 22.5 & 20.5 & 23.3 & \\
\hline & & & lower $95 \% \mathrm{Cl}$ & 20.0 & 19.7 & 16.9 & 16.0 & 20.8 & \\
\hline & & & upper $95 \% \mathrm{Cl}$ & 23.4 & 28.2 & 26.0 & 30.6 & 29.5 & \\
\hline \multirow[t]{3}{*}{$\begin{array}{l}17 \alpha, 20 \alpha \text {-dihydro- } \\
\text { progesterone }\end{array}$} & $\begin{array}{l}\text { 4-pregnen-17,20 } \alpha \text {-diol- } \\
\text { 3-one }\end{array}$ & $\mathrm{ng} / \mathrm{mL}$ & median & 38.0 & 42.4 & 39.7 & 47.9 & 40.6 & \\
\hline & & & lower $95 \% \mathrm{Cl}$ & 35.3 & 35.0 & 29.1 & 24.2 & 14.5 & \\
\hline & & & upper $95 \% \mathrm{Cl}$ & 44.8 & 55.7 & 54.2 & 54.0 & 52.6 & \\
\hline \multirow[t]{3}{*}{ 11-keto-progesterone } & $\begin{array}{l}\text { 4-pregnen-3,11,20- } \\
\text { trione }\end{array}$ & $\mathrm{ng} / \mathrm{mL}$ & median & 67.5 & 79.3 & 64.1 & 64.8 & 70.0 & a) ${ }^{*}$ \\
\hline & & & lower $95 \% \mathrm{Cl}$ & 65.5 & 71.0 & 45.5 & 54.5 & 63.4 & \\
\hline & & & upper $95 \% \mathrm{Cl}$ & 73.4 & 103.2 & 74.1 & 112.9 & 89.6 & \\
\hline Estriol (E3) & $\begin{array}{l}\text { 1,3,5(10)-estratrien- } \\
3,16 \alpha, 17 \beta \text {-triol }\end{array}$ & $\mathrm{ng} / \mathrm{mL}$ & median & 12.2 & 12.9 & 10.6 & 12.4 & 15.2 & b) ${ }^{*}$ \\
\hline & & & lower $95 \% \mathrm{Cl}$ & 11.3 & 10.5 & 8.0 & 5.8 & 9.9 & \\
\hline & & & upper $95 \% \mathrm{Cl}$ & 13.3 & 14.1 & 11.8 & 18.5 & 16.4 & \\
\hline
\end{tabular}




\section{Discussion}

$\nabla$

The importance of lipid $[8,22]$ and steroid hormone metabolism $[23,24]$ for the maintenance of pregnancy has long been known. However, the regulatory mechanisms leading to a hyperlipidemic state during pregnancy are still not fully understood. The maternal liver as a primary source of lipids and the fetoplacental unit as the most important source of steroid hormones are intimately involved. As such, we expected to see concerted changes in steroid hormone production and lipid availability. Contrary to this assumption, we observed a rather moderate correlation between lipid and steroid levels in pregnancy, suggesting that there are other factors which independently regulate both lipogenesis and steroidogenesis. Specifically, steroid and lipid hormone metabolism are compromised when affected by pathological processes. This was best observed when placental insufficiency reflected by IUGR was present, while only minor changes were observed in patients with hepatic involvement characterized by pathological liver function test, e.g. HELLP syndrome and ICP.

Life-style factors such as smoking during pregnancy contribute at least in part to metabolic changes. In line with previous publications [25] we found estradiol concentrations to be lower in the third trimester in smokers compared to non-smokers, most likely because of a reduction in placental aromatase activity [26]. Other factors that have been reported to influence maternal lipid concentrations during pregnancy are pre-pregnancy BMI and ethnic origin. Schreuder et al. found a remarkable interaction between ethnicity and cholesterol or triglyceride values throughout gestation, with the highest interdependencies found in Turkish women while in Dutch women BMI had only minor effects [27]. In our study of patients of Caucasian, predominantly German, origin, a higher pre-pregnancy BMI significantly affected HDL concentrations in the first trimester and post partum. However, significant changes could not be observed as the pregnancy progressed, suggesting that the effect of BMI on lipid values is much less than the effect caused by the pregnancy itself.

In pregnancy pathologies, the most significant differences in lipid and steroid hormone levels were observed for IUGR. The lower maternal LDL cholesterol concentrations were in agreement with those of previous reports [28]. ApoB-containing LDL particles are regarded as the main source of cholesterol for placental steroid hormone synthesis [7]. Indeed, lower steroid hormone levels of progesterone, estradiol, estriol, and allopregnanolone ( $5 \alpha$-pregnan-3 $\alpha$-ol-20-one) were found in the present study in the IUGR group compared to the CTRL group. Since cholesterol, especially LDL cholesterol, is essential for steroid hormone synthesis, the lower hormone concentrations can be regarded as secondary to low substrate levels. Levels of progesterone and allopregnanolone are highly correlated throughout pregnancy [29]. Allopregnanolone is a neuroactive metabolite of progesterone synthesized from $5 \alpha$-dihydroprogesterone by the enzyme $3 \alpha$-hydroxysteroid dehydrogenase. The potential role of neurosteroids in protecting and regulating neurodevelopmental processes in the fetal brain has been recognized recently $[30,31]$, and this finding may offer a new direction for the treatment of neurodevelopmental disorders in infants who are exposed to perinatal insults and pathologies. Hence it would be of great interest to analyze concentrations of allopregnanolone in the fetal compartment in IUGR pregnancies.

Lower $17 \beta$-estradiol concentrations have been shown to result in an increase in LDL-receptor expression in placental explant experiments [32]. Thus, our results of lower $17 \beta$-estradiol levels in patients with IUGR could explain the higher LDL-receptor mRNA expression previously observed in IUGR placentas [33]. This mechanism might be an attempt at compensation to ensure sufficient placental cholesterol supply [5]. However, since $17 \beta$-estradiol has anti-oxidative properties protecting LDL from oxidation within the placenta [34], a lower 17ß-estradiol concentration in addition to increasing LDL elimination from the maternal blood stream could subsequently lead to increased formation and accumulation of oxidized LDL particles [35]. Lower progesterone concentrations observed in IUGR contribute to the idea of lower cholesterol biosynthesis rates, either due to reduced placental function because of the toxic effects of oxidative products or as a result of a diminished concentration of native substrate, i.e. nonoxidized (LDL) cholesterol.

Recently we showed that LDL-C concentrations were reduced in women with IUGR even when they had the additional complication of PE, while women with PE but not IUGR had normal LDL levels [13]. It has been suggested that impaired placental function causes the changes in lipid profiles. It is therefore important to note that, in women with PE in whom IUGR was excluded, there were no significant changes in either lipid or steroid hormone levels apart from a 57\% higher mean 11-keto-progesterone concentration in the PE group compared to the CTRL group in the present study. This most likely reflects changes in placental $11 \beta$ HSD2 activity which primarily deactivate the 11-ketosteroid cortisol to protect the fetus from high cortisol levels [36,37].

Increased TG levels have frequently been observed in women with PE [38], but the issue is still discussed controversially [39]. In the present study we only found a tendency towards higher TG concentrations in women with PE. One explanation for the discrepant TG levels in the different studies could be the inclusion of different subtypes and cohorts of PE. Obesity is one of the predisposing factors. In our study, after the exclusion of obese patients, we found that patients with PE were only borderline overweight compared to the CTRL group. Use of the terms "placental PE" and "maternal PE" have been proposed to underline the fact that different pathomechanisms are involved at different gestational ages at the onset of disease (e.g. before 34 WoG vs. after $34 \mathrm{WoG}$ ). However, a comparison of early-onset with late-onset PE did not result in differences (data not shown). HELLP syndrome is a life-threatening condition that has been shown to affect both the placenta and the liver. Hence, massive changes in lipid profiles would have been expected. Only a few studies have evaluated patients with HELLP syndrome. Wetzka et al. observed an increase in TG concentrations in the intermediate density lipoprotein fraction [11]. We found TC levels to be lower in the HELLP syndrome group compared to the CTRL group. Since steroid hormones are not affected, lower TC concentrations may be related to impaired hepatic lipogenesis.

By contrast, higher LDL and lower HDL concentrations have been reported in the livers of women with ICP [14], a finding which was partly confirmed by our own analysis. Since these changes could already be observed before the clinical onset of disease, they are most likely a cause rather than a consequence of disease [14]. Moreover, steroid hormones may play a role in the pathophysiology. Estradiol-17 $\beta$-D-glucuronides, for example, have cholestastic properties [40]. Progesterone may play an even greater role in the pathogenesis of ICP. An increase in bile acids and AST has been observed in studies in which progesterone was given to prevent preterm birth [41]. However, in concert with the results presented here, total progesterone levels did not rise in the ICP group compared to the group with normal 
pregnancies. An excess of monosulfate and disulfate progesterone metabolites have been reported in the serum and urine of women with ICP, which may reflect impaired excretion or abnormal synthesis of these metabolites [42].

The small sample sizes, especially for the ICP and HELLP syndrome groups, limit the interpretation of our results. Moreover, blood samples were not drawn under defined nutritional conditions. This might affect TG values but should only have a limited impact on cholesterol or steroid hormones. LDL, for example, has a residence time of several days, and short-term fluctuations can be ignored [22]. In clinical practice, impairment of steroid profiles is determined based on $24 \mathrm{~h}$ urine collection, since steroid hormone production follows a circadian rhythm and blood sampling only provides a snapshot of the changes occurring during the day. Circulating concentrations of steroids also do not necessarily mimic urinary excretion of steroids, given the tissue-specific inactivation/metabolism of steroids. Nevertheless, the strength of this study is its well-characterized patient cohort and the comparisons done in our cross-sectional analysis of pregnancy-related changes observed in the longitudinal study arm. The use of GC-MS resulted in a highly specific assessment of the steroid hormones of interest, avoiding the frequently observed cross-reactivity occurring with conventional ELISA- or RIA-based measurement techniques.

In conclusion, different pregnancy pathologies are associated with characteristic lipid and steroid hormone profiles. A very pronounced difference was observed in serum estradiol and progesterone concentrations in IUGR pregnancies. Interestingly, although the liver is the primary source of lipid synthesis, placental insufficiency is more likely to affect lipid and steroid hormone metabolism than pregnancy-related liver disease.

Our results suggest that more targeted research into lipid and steroid hormone metabolism in pregnancy pathologies, with a special focus on IUGR, is indicated.

\section{Conflict of Interest}

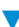

None.

\section{References}

1 Lobo RA, Skinner JB, Lippman JS et al. Plasma lipids and desogestrel and ethinyl estradiol: a meta-analysis. Fertil Steril 1996; 65: 1100-1109

2 Al-Azzawi F, Wahab M, Sami S et al. Randomized trial of effects of estradiol in combination with either norethisterone acetate or trimegestone on lipids and lipoproteins in postmenopausal women. Climacteric 2004; 7: 292-300

3 Walsh BW, Schiff I, Rosner B et al. Effects of postmenopausal estrogen replacement on the concentrations and metabolism of plasma lipoproteins. N Engl J Med 1991; 325: 1196-1204

4 Homma H, Kurachi H, Nishio Y et al. Estrogen suppresses transcription of lipoprotein lipase gene. Existence of a unique estrogen response element on the lipoprotein lipase promoter. J Biol Chem 2000; 275 : 11404-11411

5 Grimes RW, Pepe GJ, Albrecht ED. Regulation of human placental trophoblast low-density lipoprotein uptake in vitro by estrogen. J Clin Endocrinol Metab 1996; 81: 2675-2679

6 Baulieu EE, Dray F. CONVERSION OF H3-DEHYDROISOANDROSTERONE (3 BETA-HYDROXY-DELTA5-ANDROSTEN-17-ONE) SULFATE TO H3ESTROGENS IN NORMAL PREGNANT WOMEN. J Clin Endocrinol Metab 1963; 23: 1298-1301

7 Rabe T, Kalbfleisch H, Haun A et al. Influence of human lipoproteins on the progesterone synthesis of human term placenta in organ culture. Biol Res Pregnancy Perinatol 1983; 4: 75-83
8 Sattar N, Greer IA, Louden J et al. Lipoprotein subfraction changes in normal pregnancy: threshold effect of plasma triglyceride on appearance of small, dense low density lipoprotein. J Clin Endocrinol Metab 1997; 82: 2483-2491

9 Pecks U, Tillmann D, Ernst S et al. Anti-oxidized low-density lipoprotein (oxLDL) antibody levels are not related to increasing circulating oxLDL concentrations during the course of pregnancy. Am J Reprod Immunol 2012; 68: 345-352

10 Seiler E, Woitke AK, Schollberg K et al. [Correlation of progesterone and apoprotein B in pregnancy]. Zentralbl Gynakol 1988; 110: 1340-1344

11 Wetzka B, Winkler K, Kinner $M$ et al. Altered lipid metabolism in preeclampsia and HELLP syndrome: links to enhanced platelet reactivity and fetal growth. Semin Thromb Hemost 1999; 25: 455-462

12 Winkler K, Wetzka B, Hoffmann MM et al. Triglyceride-rich lipoproteins are associated with hypertension in preeclampsia. J Clin Endocrinol Metab 2003; 88: 1162-1166

13 Pecks $U$, Caspers $R$, Schiessl B et al. The evaluation of the oxidative state of low-density lipoproteins in intrauterine growth restriction and preeclampsia. Hypertens Pregnancy 2012; 31: 156-165

14 Dann AT, Kenyon AP, Wierzbicki AS et al. Plasma lipid profiles of women with intrahepatic cholestasis of pregnancy. Obstet Gynecol 2006; 107: 106-114

15 Hertig A, Liere P, Chabbert-Buffet $N$ et al. Steroid profiling in preeclamptic women: evidence for aromatase deficiency. Am J Obstet Gynecol 2010; 203: 477.e1-477.e9

16 Abu-Hayyeh S, Papacleovoulou G, Lövgren-Sandblom A et al. Intrahepatic cholestasis of pregnancy levels of sulfated progesterone metabolites inhibit farnesoid $\mathrm{X}$ receptor resulting in a cholestatic phenotype. Hepatology 2013; 57: 716-726

17 Rath W, Faridi A, Dudenhausen JW. HELLP syndrome. J Perinat Med 2000; 28: 249-260

18 Reimer T, Rohrmann H, Stubert J et al. Angiogenic factors and acutephase proteins in serum samples of preeclampsia and HELLP patients: a matched-pair analysis. J Matern Fetal Neonatal Med 2013; 26: 263269

19 Mays JK. The active management of intrahepatic cholestasis of pregnancy. Curr Opin Obstet Gynecol 2010; 22: 100-103

20 Parker CR jr., Atkinson MW, Owen J et al. Dynamics of the fetal adrenal, cholesterol, and apolipoprotein B responses to antenatal betamethasone therapy. Am J Obstet Gynecol 1996; 174: 562-565

21 Vögeli I, Jung HH, Dick B et al. Evidence for a role of sterol 27-hydroxylase in glucocorticoid metabolism in vivo. J Endocrinol 2013; 219: 119129

22 Winkler K, Wetzka B, Hoffmann MM et al. Low density lipoprotein (LDL) subfractions during pregnancy: accumulation of buoyant LDL with advancing gestation. J Clin Endocrinol Metab 2000; 85: 4543-4550

23 Peter M, Dörr HG, Sippell WG. Changes in the concentrations of dehydroepiandrosterone sulfate and estriol in maternal plasma during pregnancy: a longitudinal study in healthy women throughout gestation and at term. Horm Res 1994; 42: 278-281

24 Dörr HG, Heller A, Versmold HT et al. Longitudinal study of progestins, mineralocorticoids, and glucocorticoids throughout human pregnancy. J Clin Endocrinol Metab 1989; 68: 863-868

25 Bartels I, Hoppe-Sievert B, Bockel B et al. Adjustment formulae for maternal serum alpha-fetoprotein, human chorionic gonadotropin, and unconjugated oestriol to maternal weight and smoking. Prenat Diagn 1993; 13: 123-130

26 Kitawaki J, Inoue S, Tamura T et al. Cigarette smoking during pregnancy lowers aromatase cytochrome P-450 in the human placenta. J Steroid Biochem Mol Biol 1993; 45: 485-491

27 Schreuder YJ, Hutten BA, van Eijsden $M$ et al. Ethnic differences in maternal total cholesterol and triglyceride levels during pregnancy: the contribution of demographics, behavioural factors and clinical characteristics. Eur J Clin Nutr 2011; 65: 580-589

28 Sattar N, Greer IA, Galloway PJ et al. Lipid and lipoprotein concentrations in pregnancies complicated by intrauterine growth restriction. J Clin Endocrinol Metab 1999; 84: 128-130

29 Gilbert Evans SE, Ross LE, Sellers EM et al. 3alpha-reduced neuroactive steroids and their precursors during pregnancy and the postpartum period. Gynecol Endocrinol 2005; 21: 268-279

30 Yawno T, Hirst JJ, Castillo-Melendez M et al. Role of neurosteroids in regulating cell death and proliferation in the late gestation fetal brain. Neuroscience 2009; 163: 838-847 
31 Kelleher MA, Palliser HK, Walker DW et al. Sex-dependent effect of a low neurosteroid environment and intrauterine growth restriction on fetal guinea pig brain development. J Endocrinol 2010; 208: 301-309

32 Shanker YG, Shetty UP, Rao AJ. Regulation of low density lipoprotein receptor mRNA levels by estradiol 17beta and chorionic gonadotropin in human placenta. Mol Cell Biochem 1998; 187: 133-139

33 Stepan $H$, Faber $R$, Walther $T$. Expression of low density lipoprotein receptor messenger ribonucleic acid in placentas from pregnancies with intrauterine growth retardation. Br J Obstet Gynaecol 1999; 106: $1221-1222$

34 Zhu XD, Bonet B, Knopp RH. 17beta-Estradiol, progesterone, and testosterone inversely modulate low-density lipoprotein oxidation and cytotoxicity in cultured placental trophoblast and macrophages. Am J Obstet Gynecol 1997; 177: 196-209

35 Pecks $U$, Rath W, Caspers $R$ et al. Oxidatively modified LDL particles in the human placenta in early and late onset intrauterine growth restriction. Placenta 2013; 34: 1142-1149

36 Kosicka K, Siemiątkowska A, Krzyścin $M$ et al. Glucocorticoid metabolism in hypertensive disorders of pregnancy: analysis of plasma and urinary cortisol and cortisone. PLoS One 2015; 10: e0144343
37 Causevic M, Mohaupt M. 11ß-Hydroxysteroid dehydrogenase type 2 in pregnancy and preeclampsia. Mol Aspects Med 2007; 28: 220-226

38 Gallos ID, Sivakumar K, Kilby MD et al. Pre-eclampsia is associated with, and preceded by, hypertriglyceridaemia: a meta-analysis. BJOG 2013; 120: 1321-1332

39 Hentschke MR, Poli-de-Figueiredo CE, da Costa BEP et al. Is the atherosclerotic phenotype of preeclamptic placentas due to altered lipoprotein concentrations and placental lipoprotein receptors? Role of a small-for-gestational-age phenotype. J Lipid Res 2013; 54: 2658-2664

40 Davis RA, Kern F, Showalter R et al. Alterations of hepatic $\mathrm{Na}+, \mathrm{K}+-$ atpase and bile flow by estrogen: effects on liver surface membrane lipid structure and function. Proc Natl Acad Sci U S A 1978; 75: 4130-4134

41 Benifla JL, Dumont $M$, Levardon $M$ et al. [Effects of micronized natural progesterone on the liver during the third trimester of pregnancy]. Contracept Fertil Sex 1997; 25: 165-169

42 Meng L, Reyes $H$, Axelson $M$ et al. Progesterone metabolites and bile acids in serum of patients with intrahepatic cholestasis of pregnancy: Effect of ursodeoxycholic acid therapy. Hepatology 1997; 26: 1573-1579 